\title{
The asymptotic zero distribution of sections and tails of classical Lindelöf functions
}

\author{
Iossif Ostrovskii ${ }^{* 1}$ and Natalya Zheltukhina ${ }^{* * 1}$ \\ ${ }^{1}$ Bilkent University, Department of Mathematics, 06800 Bilkent, Ankara, Turkey
}

Received 16 November 2006, revised 19 June 2008, accepted 29 August 2008

Published online 18 March 2010

Key words Lindelöf functions, sections, tails, zero distribution

MSC (2000) 30D15, 30C15

We study the asymptotic (as $n \rightarrow \infty$ ) zero distribution of

$$
I_{n}\left(z, \mu, \Gamma_{\lambda}\right)=(1-\mu) s_{n}\left(z, \Gamma_{\lambda}\right)-\mu t_{n+1}\left(z, \Gamma_{\lambda}\right),
$$

where $\mu \in \mathbb{C}, s_{n}$ is $n^{\text {th }}$ section, $t_{n}$ is $n^{\text {th }}$ tail of the power series of classical Lindelöf function $\Gamma_{\lambda}$ of order $\lambda$. Our results generalize the results by A. Edrei, E. B. Saff, and R. S. Varga for the case $\mu=0$.

(c) 2010 WILEY-VCH Verlag GmbH \& Co. KGaA, Weinheim

\section{Introduction}

For a transcendental entire function

$$
f(z)=\sum_{k=0}^{\infty} a_{k} z^{k}, \quad a_{0}>0
$$

denote by

$$
s_{n}(z, f)=\sum_{k=0}^{n} a_{k} z^{k} \text { and } t_{n}(z, f)=\sum_{k=n}^{\infty} a_{k} z^{k}
$$

its $n^{\text {th }}$ section and $n^{\text {th }}$ tail respectively.

For some widely applicable concrete entire functions (such as the exponential function, the trigonometric functions and some others) elegant and sharp asymptotics (as $n \rightarrow \infty)$ for zeros of $s_{n}(z, f)$ and $t_{n}(z, f)$ were obtained by G. Szegö [8], J. Dieudonné [1], P. C. Rosenbloom [7] and others. In the work of A. Edrei, E. B. Saff, and R. S. Varga [2] these asymptotics for zeros of $s_{n}(z, f)$ were extended to the Mittag-Leffler functions and to $\mathcal{L}$-functions.

Recall that $F(z)$ is called an $\mathcal{L}$-function if it satisfies the following two conditions.

(A) The function $F(z)$ is entire of order $\lambda(0<\lambda<1)$ and all its zeros are real and negative:

$F(z)=F(0) \prod_{k=1}^{\infty}\left(1+\frac{z}{x_{k}}\right)=\sum_{j=0}^{\infty} a_{j} z^{j}, \quad$ where $\quad 0<x_{k}, \quad \sum_{k=1}^{\infty} x_{k}^{-1}<+\infty, \quad F(0)>0$

(B) Along the positive axis

$$
\ln F(r)=\ln M(r, F)=B_{1} r^{\lambda}(1+o(1)), \quad B_{1}>0, \quad r \longrightarrow \infty .
$$

* e-mail: iossif@fen.bilkent.edu.tr, Phone: +90 312290 2747, Fax: +90 3122664579 ,

** Corresponding author: e-mail: natalya@fen.bilkent.edu.tr, Phone: +90 3122902465 , Fax: +90 3122664579 , 
G. Szegö in [8] considered a more general problem of the asymptotic distribution of the zeros of the linear combination

$$
I_{n}(z, \mu, f)=(1-\mu) s_{n}(z, f)-\mu t_{n+1}(z, f)
$$

when $\mu \in \mathbb{C}$. Evidently, $I_{n}(z, 0, f)=s_{n}(z, f)$ and $I_{n}(z, 1, f)=-t_{n+1}(z, f)$. G. Szegö in [8] proved a remarkable theorem related to the asymptotic behavior of the roots of the equation

$$
I_{n}\left(z, \mu, e^{z}\right)=0 \text {. }
$$

It was discovered by G. Szegö that the set of all zeros of

$$
I_{n}\left(z, \mu, e^{z}\right), \quad \mu \neq 0,1,
$$

is approximately equal to $\left\{n z:\left|z e^{1-z}\right|=1\right\}$, the set of all zeros of $s_{n}\left(z, e^{z}\right)$ is approximately equal to $\left\{n z:\left|z e^{1-z}\right|=1,|z| \leq 1\right\}$, the set of all zeros of $t_{n}\left(z, e^{z}\right)$ is approximately equal to $\left\{n z:\left|z e^{1-z}\right|=1\right.$, $|z| \geq 1\}$.

A survey of investigations prior to 1997 on several aspects of the distribution of zeros of sections and tails is given by I. V. Ostrovskii in [6].

In [9], the zero distribution of linear combinations (1.5) of Mittag-Leffler functions was considered. The results obtained in [9] extend some results of A. Edrei, E. B. Saff, and R. S. Varga [2] on the zero distribution of sections $s_{n}(z, f)$ of Mittag-Leffler functions.

The following problem seems to be of interest. Is it possible to extend the results of A. Edrei, E.B. Saff and R.S. Varga [2] on the zero distribution of sections $s_{n}(z, f)$ of $\mathcal{L}$-functions to the zero distribution of linear combinations (1.5) of $\mathcal{L}$-functions?

Below we present the main result of [2] on $\mathcal{L}$-functions (see [2], p. 21).

Theorem A. Let $F(z)$ be an $\mathcal{L}$-function of order $\lambda$.

I. Define the sequence $\left\{R_{m}\right\}_{m}$ by the conditions

$$
a\left(R_{m}\right)=m \quad(m=1,2,3, \ldots), \quad \text { where } \quad a(r)=r \frac{F^{\prime}(r)}{F(r)} .
$$

Let $\operatorname{erfc}(\zeta)$ denote the complementary error function

$$
\operatorname{erfc}(\zeta)=1-\frac{2}{\sqrt{\pi}} \int_{0}^{\zeta} e^{-v^{2}} d v=\frac{2}{\sqrt{\pi}} \int_{\zeta}^{\infty} e^{-v^{2}} d v .
$$

Then, if $\zeta$ is an auxiliary complex variable, we have

$$
\frac{s_{m}\left(R_{m}\left(1+\left(\frac{2}{\lambda m}\right)^{1 / 2} \zeta\right), F\right)}{F\left(R_{m}\right)\left(1+\left(\frac{2}{\lambda m}\right)^{1 / 2} \zeta\right)^{m}} \longrightarrow \frac{1}{2} \exp \left(\zeta^{2}\right) \operatorname{erfc}(\zeta),
$$

uniformly on every compact set of the $\zeta$-plane.

II. With every given $\phi(0<|\phi|<\pi)$ it is possible to associate a real sequence $\left\{\sigma_{m}(\phi)\right\}$ such that

$$
\lim _{m \rightarrow \infty} \sigma_{m}(\phi)=\sigma(\phi)
$$

where $\sigma=\sigma(\phi)$ is the unique solution in $(0,1)$ of the equation

(i)

$$
\sigma^{\lambda} \cos (\phi \lambda)-1-\lambda \ln \sigma=0
$$

(ii) write

$$
\xi_{m}=\xi_{m}(\phi)=\sigma_{m}(\phi) e^{i \phi}, \quad \xi=\sigma(\phi) e^{i \phi}, \quad L_{m}=(2 \pi \lambda m)^{1 / 2} \xi_{m}^{-m}\left\{F\left(R_{m}\right)\right\}^{-1} ;
$$


then the polynomials in $\zeta$

$$
L_{m} s_{m}\left(R_{m} \xi_{m}\left(1+\frac{\zeta}{m\left(1-\xi^{\lambda}\right)}\right)\right)
$$

are uniformly bounded on every compact set of the $\zeta$-plane.

III. Every limit function of the polynomials in (1.8) is of the form

$$
\exp \left(\frac{\zeta}{1-\xi^{\lambda}}\right)\left\{e^{i \chi} e^{-\zeta}-\frac{\xi}{1-\xi}\right\}=Z_{\chi}(\zeta),
$$

where the real quantity $\chi$ may depend on the particular sequence of integers through which $m \rightarrow+\infty$.

For any $\mathcal{L}$-function $F$ of order $\lambda$, let $\mathcal{M}_{n}(\mu, F), \mu \in \mathbb{C}$, be the set of all roots of the equation

$$
I_{n}\left(R_{n} z, \mu, F\right)=0 \text {, }
$$

where

$$
I_{n}\left(R_{n} z, \mu, F\right)=(1-\mu) s_{n}\left(R_{n} z, F\right)-\mu t_{n+1}\left(R_{n} z, F\right) .
$$

In particular, $\mathcal{M}_{n}(0, F)\left(\mathcal{M}_{n-1}(1, F)\right)$ coincides with the zero set of $s_{n}\left(R_{n} z, F\right)\left(t_{n}\left(R_{n-1} z, F\right)\right)$. Define $\mathcal{M}(\mu, F)$ to be the set of all accumulation points of $\bigcup_{n=1}^{\infty} \mathcal{M}_{n}(\mu, F)$.

It follows from Theorem A, parts II and III, that

$$
\left\{z=\sigma e^{i \phi}: \sigma^{\lambda} \cos (\phi \lambda)-1-\lambda \ln \sigma=0,0<\sigma<1,0<|\phi|<\pi\right\} \subset \mathcal{M}(0, F)
$$

for any $\mathcal{L}$-function $F$.

The following problem seems to be of interest. Does the embedding (1.9) remain in force if we replace $\mathcal{M}(0, F)$ by $\mathcal{M}(\mu, F)$ when $\mu \in \mathbb{C}$ ? In the present paper we study the zero distribution of the linear combination $I_{n}\left(R_{n} z, \mu, F\right)$ of the Lindelöf classical functions

$$
\Gamma_{\lambda}(z)=\prod_{n=1}^{\infty}\left(1+\frac{z}{n^{1 / \lambda}}\right), \quad 0<\lambda<1,
$$

and show that for Lindelöf classical functions (not arbitrary $\mathcal{L}$-function) the embedding (1.9) can be extended to all $\mu$ in $\mathbb{C}$, and moreover, changed to equality. To our knowledge, for arbitrary $\mathcal{L}$-function the answer to the above question is still open.

\section{Main curves and regions}

To formulate the main result of the paper we need to introduce some curves and regions. For any $\lambda$ satisfying $0<\lambda<1$, and $h$, being sufficiently small, denote

$$
S(\lambda, h)=\left\{z=r e^{i \phi}: r^{\lambda} \cos (\lambda \phi)-\lambda \ln r-1=h,|\phi| \leq \pi\right\} .
$$

Clearly, $S(\lambda, h)$ is symmetric with respect to the $x$-axis. We have, if $z=r e^{i \phi} \in S(\lambda, h)$,

$$
\cos (\lambda \phi)=g(r, h)=\frac{1+h+\lambda \ln r}{r^{\lambda}} .
$$

Since $\frac{d g(r, h)}{d r}=-\frac{\lambda(h+\lambda \ln r)}{r^{\lambda+1}}$, then $g(r, h)$ increases when $r \in\left(0, e^{-h / \lambda}\right)$ and decreases when $r \in\left(e^{-h / \lambda}, \infty\right)$. We give rough shapes of the curves $S(\lambda, h)$ in three different cases (when $h=0, h>0$ and $h<0$ ) in Fig. 1, Fig. 2 and Fig. 3.

Let us fix constants $\lambda$ and $h, 0<\lambda<1, h \geq 0$. Note that the curve $S(\lambda, h)$ divides the complex plane $\mathbb{C}$ into three different regions. Denote by $I_{h}$ and $I_{h}$ two of these three regions. Namely, let $I_{h}$ be the region containing $z=0$ and let $I I_{h}$ be the region that contains neither $z=0$ nor -1 . Curve $S(\lambda,-h)$ divides the complex plane $\mathbb{C}$ into two different regions. Denote by $I I I_{h}$ that region that does not contain $z=0$. We give rough sketches of the regions $I_{h}, I I_{h}$ and $I I I_{h}$ in Fig.4.

If $0<\varepsilon_{1}<\pi$, we define

$$
\Delta=\Delta\left(\varepsilon_{1}\right)=\left\{z=r e^{i \phi}:|\phi| \leq \pi-\varepsilon_{1}, r>0\right\} .
$$



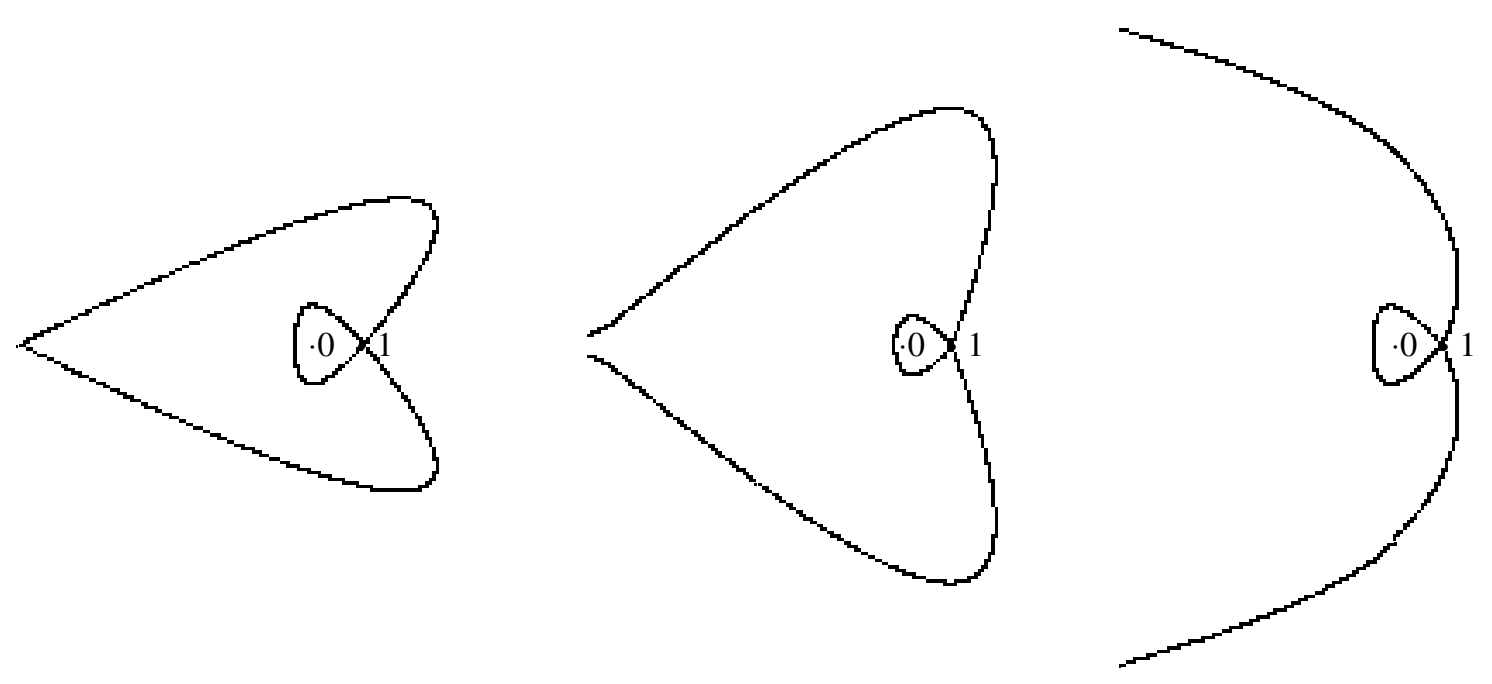

Fig. 1 Curves $S(\lambda, 0)$ for $0<\lambda<1 / 2, \lambda=1 / 2$ and $1 / 2<\lambda<1$ respectively.
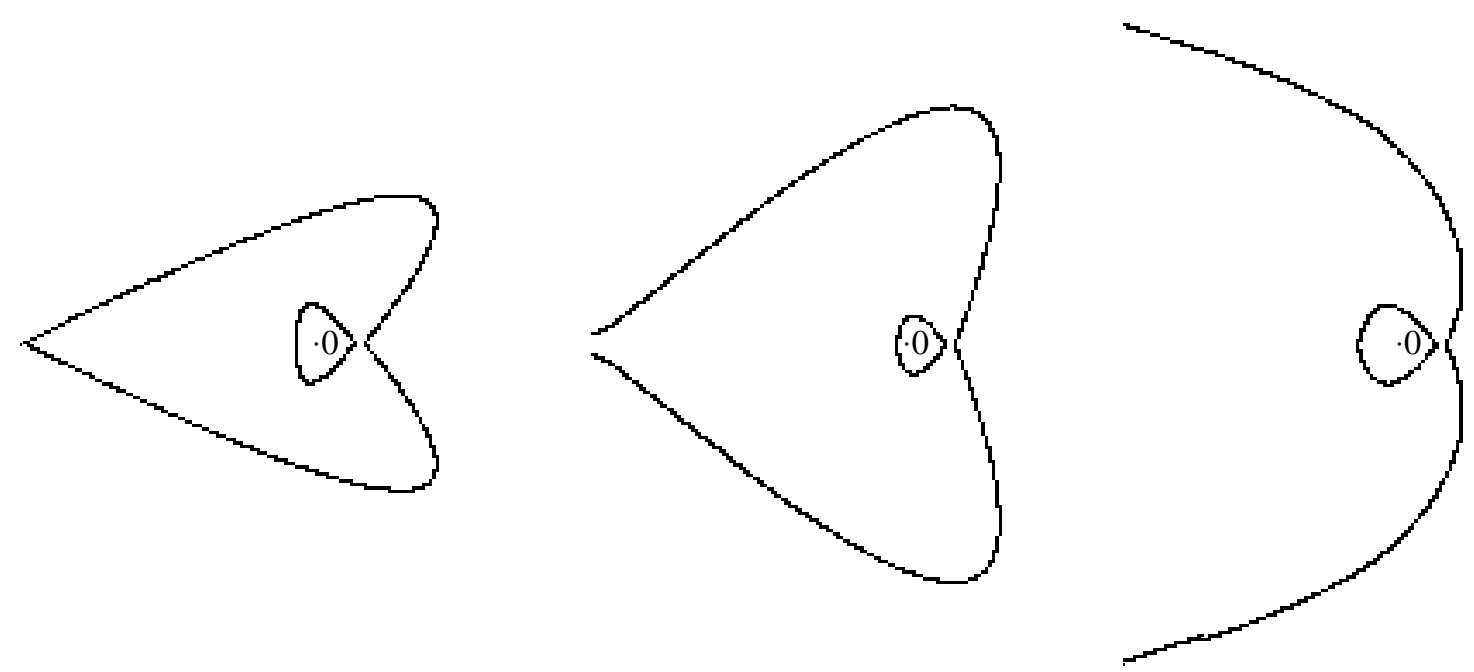

Fig. 2 Curves $S(\lambda, h)$ with $h>0$ for $0<\lambda<1 / 2, \lambda=1 / 2$ and $1 / 2<\lambda<1$ respectively

\section{Results}

The first theorem we prove shows regions where zeros of $I_{m}\left(R_{m} w, \mu, \Gamma_{\lambda}\right)$ may be.

Theorem 3.1 Let $\Gamma_{\lambda}(z)$ be a Lindelöf classical function of order $\lambda(0<\lambda<1)$. Suppose that $\mu \neq 0$. Then, if $\delta, \varepsilon_{1}$ and h are sufficiently small positive constants, $I_{m}\left(R_{m} w, \mu, \Gamma_{\lambda}\right)$ does not vanish in $\left(I_{0} \cup I I_{0} \cup I I I_{h}\right) \cap \Delta$, for all sufficiently large $m$.

Theorem 3.1 implies that the zeros of $I_{m}\left(R_{m} w, \mu, \Gamma_{\lambda}\right)$ may lie only in the vicinity of the curve $S(\lambda, 0)$ and the ray $\arg z=\pi$. The proof of Theorem 3.1 is given in Section 5. The case $\mu=0$ was studied in [2] not only for the classical Lindelöf function $\Gamma_{\lambda}(z)$ but for any $\mathcal{L}$-function (see Theorem A above).

We define

$$
\mathcal{M}_{*}\left(\mu, \Gamma_{\lambda}\right)=\mathcal{M}\left(\mu, \Gamma_{\lambda}\right) \backslash\{z: \arg z=\pi\} .
$$

The following remark is a corollary of Theorem 3.1. 

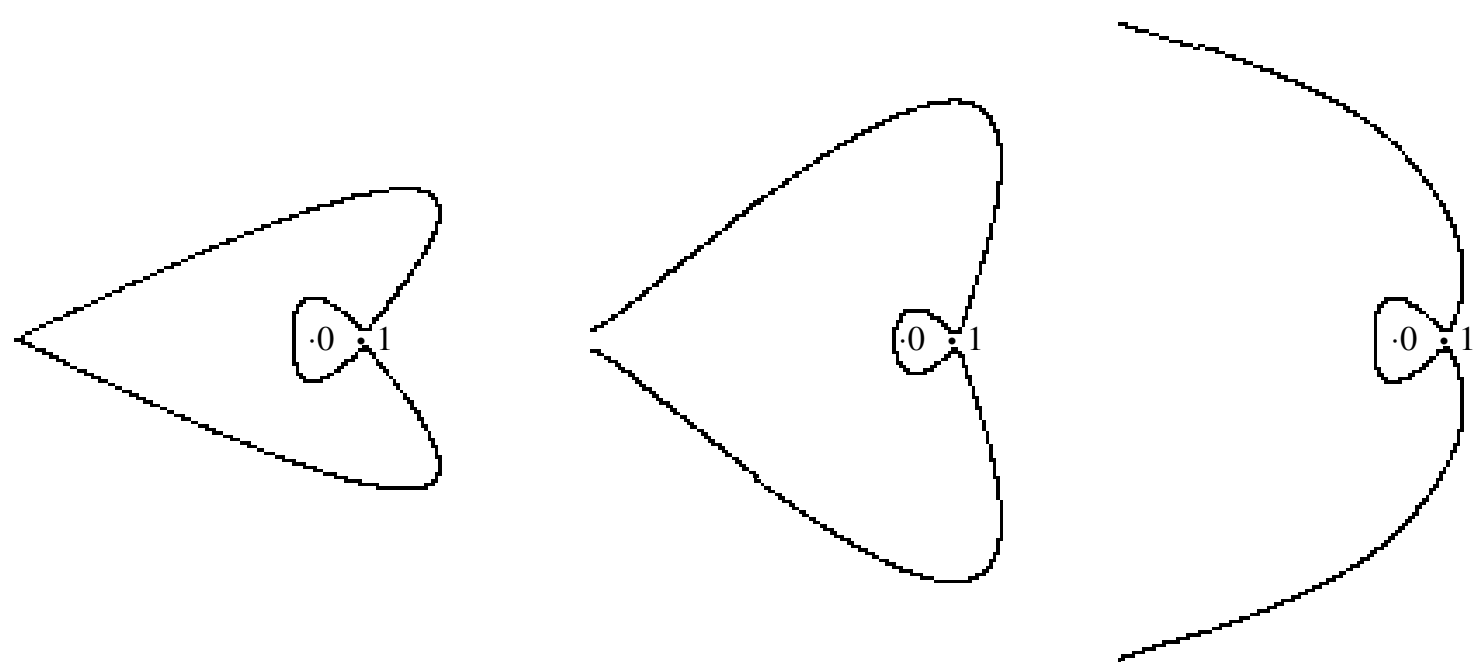

Fig. 3 Curves $S(\lambda, h)$ with $h<0$ for $0<\lambda<1 / 2, \lambda=1 / 2$ and $1 / 2<\lambda<1$ respectively
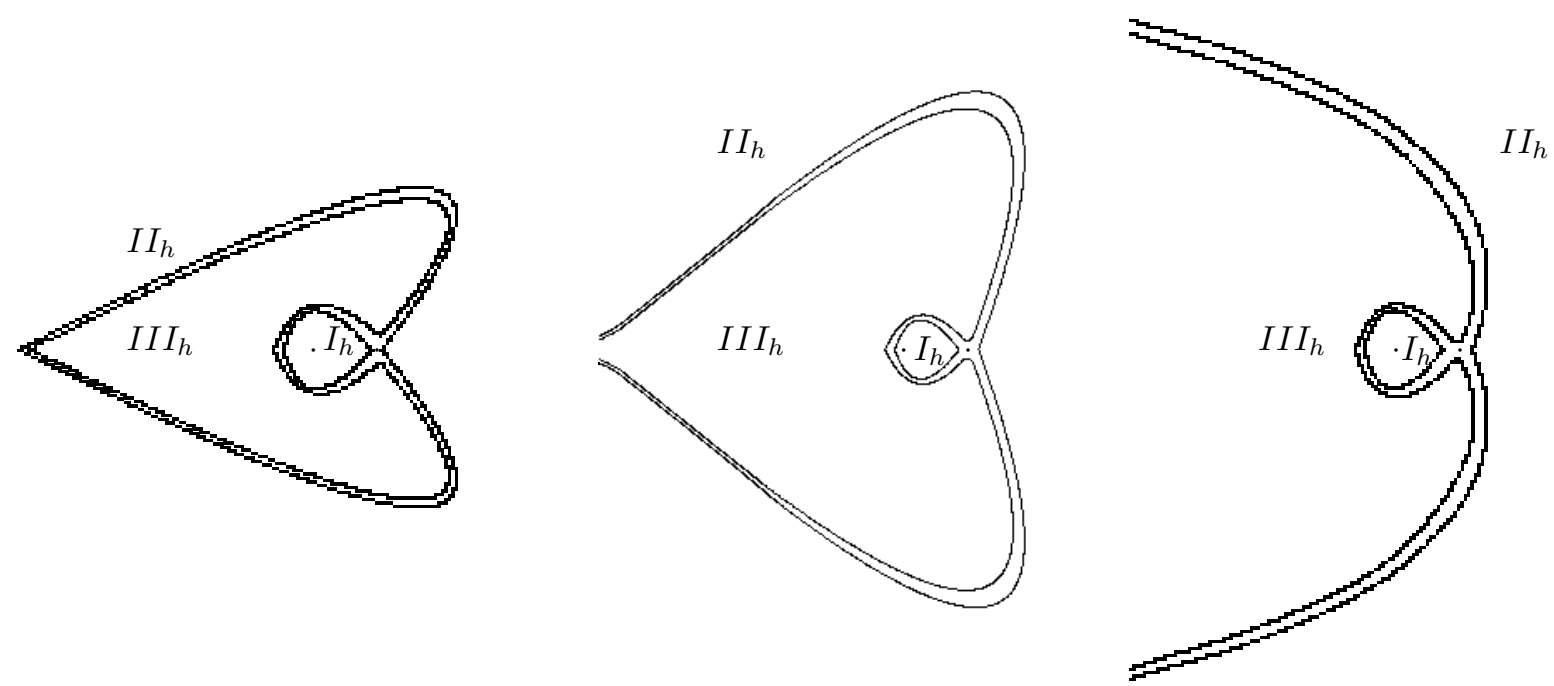

Fig. 4 Regions $I_{h}, I I_{h}$ and $I I I_{h}$ for $0<\lambda<1 / 2, \lambda=1 / 2$ and $1 / 2<\lambda<1$ respectively

\section{Remark 3.2}

$$
\mathcal{M}_{*}\left(\mu, \Gamma_{\lambda}\right) \subset S(\lambda, 0) .
$$

The next theorem shows that each point on the curve $S(\lambda, 0)$ is an accumulation point of zeros of $I_{m}\left(R_{m} z, \mu, \Gamma_{\lambda}\right)$ when $\mu \in \mathbb{C} \backslash\{0,1\}$.

Theorem 3.3 Let $\xi=\xi(\phi)=|\xi| e^{i \phi}, 0<|\phi|<\pi$, be a fixed point on the curve $S(\lambda, 0)$. We define $\tau=|\xi|^{\lambda} \sin (\lambda \phi)-\lambda \phi$, and let the sequences $\left\{\tau_{m}\right\}_{m=1}^{\infty}$ and $\left\{\varepsilon_{m}(\zeta)\right\}_{m=1}^{\infty}$ be defined by the conditions

$$
\tau_{m} \equiv \frac{\tau}{\lambda} m(\bmod \quad 2 \pi), \quad-\pi<\tau_{m} \leq \pi
$$

and

$$
\varepsilon_{m}(\zeta)=\frac{\log m}{2\left(1-\xi^{\lambda}\right) m}-\frac{\zeta-i \tau_{m}}{\left(1-\xi^{\lambda}\right) m}
$$


Then, as $m \rightarrow \infty$,

$$
\begin{aligned}
& I_{m}\left(R_{m} \xi\left(1+\varepsilon_{m}(\zeta)\right), \mu, \Gamma_{\lambda}\right)\left(\frac{2 m^{1+\lambda} \sin (\pi \lambda)}{\lambda^{1-\lambda} e}\right)^{\frac{1}{2 \lambda}} \frac{(2 \pi)^{\frac{1}{2}}}{\xi^{m}\left(1+\varepsilon_{m}(\zeta)\right)^{m} e^{\frac{m}{\lambda}}}
\end{aligned}
$$

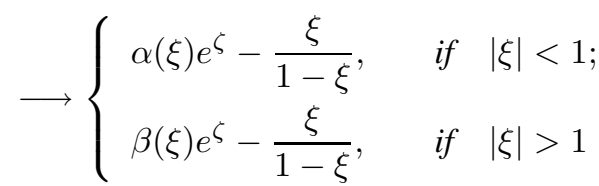

uniformly on every compact set of the $\zeta$-plane, where

$$
\alpha(\xi)=(1-\mu)\left(\frac{2 \pi \lambda}{\xi}\right)^{\frac{1}{2}} e^{\frac{\xi^{\lambda}-1}{2 \lambda}}, \quad \beta(\xi)=-\mu\left(\frac{2 \pi \lambda}{\xi}\right)^{\frac{1}{2}} e^{\frac{\xi^{\lambda}-1}{2 \lambda}} .
$$

The proof of Theorem 3.3 is given in Section 6. To prove Theorem 3.3 we repeat the proof of Theorem 2 from [2] with slight modifications.

The next result is a corollary of Theorems 3.1 and 3.3.

Corollary 3.4 One has:

(i) $\mathcal{M}_{*}\left(0, \Gamma_{\lambda}\right)=S(\lambda, 0) \cap\{z:|z| \leq 1\}$,

(ii) $\mathcal{M}_{*}\left(1, \Gamma_{\lambda}\right)=S(\lambda, 0) \cap\{z:|z| \geq 1\}$,

(iii) $\mathcal{M}_{*}\left(\mu, \Gamma_{\lambda}\right)=S(\lambda, 0)$ for $\mu \neq 0,1$.

The next result shows how quickly the zeros of $I_{m}\left(R_{m} w, \mu, F\right)$ approach the point $w=1$ for arbitrary $\mathcal{L}$-function $F$.

Theorem 3.5 Let $F(z)$ be an $\mathcal{L}$-function of order $\lambda(0<\lambda<1)$. Then, as $m \rightarrow \infty$,

$$
\frac{I_{m}\left(R_{m}\left(1+\left(\frac{2}{\lambda m}\right)^{1 / 2} \zeta\right), \mu, F\right)}{F\left(R_{m}\right)\left(1+\left(\frac{2}{\lambda m}\right)^{1 / 2} \zeta\right)^{m}} \longrightarrow \exp \left(\zeta^{2}\right)\left(\frac{\operatorname{erfc}(\zeta)}{2}-\mu\right)
$$

uniformly on every compact set of the $\zeta$-plane.

Theorem 3.5 can be viewed as an extension of part I of Theorem A and is an easy corollary of part I of Theorem A. The proof of Theorem 3.5 is given in Section 7 .

\section{Preliminaries}

Let the functions $F(z)$ and $\Gamma_{\lambda}(z)$ be given by (1.3) and (1.10) respectively. We mention without proof properties of the functions $F(z)$ and $\Gamma_{\lambda}(z)$ which the reader can find in [2], [3] and [4].

1) It is known that (see [2], p. 90)

$$
\ln F(z)=B_{1} z^{\lambda}(1+\eta(z))
$$

and

$$
z \frac{F^{\prime}(z)}{F(z)}=B_{1} \lambda z^{\lambda}(1+\eta(z))
$$

where $\eta(z) \rightarrow 0$ uniformly in $\Delta$, as $z \rightarrow \infty$.

Also (see [3], p. 158)

$$
\Gamma_{\lambda}(z)=\frac{e^{\frac{\pi z^{\lambda}}{\sin \pi \lambda}+\nu(z)}}{z^{\frac{1}{2}}(2 \pi)^{\frac{1}{2 \lambda}}}
$$


where $z \nu(z)$ is uniformly bounded in $\Delta$, as $z \rightarrow \infty$.

2) Using (4.3) we can easily calculate the indicator function of the classical Lindelöf function $\Gamma_{\lambda}$ (see [5], p. 53). It is

$$
h_{\Gamma_{\lambda}}(\theta)=\frac{\pi}{\sin \pi \lambda} \cos \lambda \theta, \quad-\pi<\theta<\pi
$$

Since the indicator function of an entire function of finite order and finite type is a continuous function then

$$
h_{\Gamma_{\lambda}}(\theta)=\frac{\pi}{\sin \pi \lambda} \cos \lambda \theta, \quad \theta \in[-\pi, \pi] .
$$

It follows (see [5], p. 56) that

$$
\left|\Gamma_{\lambda}\left(r e^{i \theta}\right)\right|<e^{\left(\frac{\pi \cos \lambda \theta}{\sin \pi \lambda}+\alpha\right) r^{\lambda}}
$$

for all $r>r(\alpha)$ and when $\theta \in[-\pi, \pi]$, where $\alpha$ is sufficiently small.

3) Let the sequence $\left\{R_{m}\right\}_{m}$ be defined by conditions (1.6). Then (see [2], p. 93)

$$
R_{m}=\left\{\frac{m}{B_{1} \lambda}\right\}^{1 / \lambda}(1+o(1)), \quad m \longrightarrow \infty
$$

and

$$
a_{m} R_{m}^{m}=\frac{F\left(R_{m}\right)}{(2 \pi \lambda m)^{1 / 2}}(1+o(1)), \quad m \longrightarrow \infty
$$

Using (1.6) and (4.3), for $\Gamma_{\lambda}(z)$ we have,

$$
\begin{aligned}
& R_{m}^{\lambda}=\frac{(m+(1 / 2)) \sin (\pi \lambda)}{\pi \lambda}+o(1), \quad m \longrightarrow \infty, \\
& R_{m}^{1 / 2}=\left(\frac{m \sin (\pi \lambda)}{\pi \lambda}\right)^{1 /(2 \lambda)}(1+o(1)), \quad m \longrightarrow \infty .
\end{aligned}
$$

It follows from (4.3), (4.7) and (4.8) that

$$
\Gamma_{\lambda}\left(R_{m} w\right)=\frac{e^{\frac{m w^{\lambda}}{\lambda}}}{m^{\frac{1}{2 \lambda}}}\left(\frac{\lambda e^{w^{\lambda}}}{2 w^{\lambda} \sin (\pi \lambda)}\right)^{\frac{1}{2 \lambda}}(1+o(1)), \quad m \longrightarrow \infty .
$$

It follows from (4.6) and (4.9) that for $\Gamma_{\lambda}(z)$ we have

$$
a_{m} R_{m}=\frac{e^{\frac{m}{\lambda}}}{(2 \pi)^{\frac{1}{2}}}\left(\frac{\lambda^{1-\lambda} e}{2 m^{1+\lambda} \sin (\pi \lambda)}\right)^{\frac{1}{2 \lambda}}(1+o(1)), \quad m \longrightarrow \infty
$$

4) Let $w$ satisfy $|w-1| \leq \eta<1 / 2$. Then (see [2], p. 96), as $m \rightarrow \infty$,

$F\left(R_{m} w\right)=F\left(R_{m}\right) \exp \left\{(w-1) m+\frac{(w-1)^{2}}{2} m(\lambda-1+o(1))+(w-1)^{3} m \eta(m, w)\right\}$,

where the sequence $\{\eta(m, w)\}_{m}$ is uniformly bounded in $\{w:|w-1| \leq \eta\}$. 


\section{Proof of Theorem 3.1}

To prove Theorem 3.1 we will find the asymptotic behavior of $I_{m}\left(R_{m} w, \mu, \Gamma_{\lambda}\right)$ in regions $I_{h}, I I_{h}$ and $I I I_{h}$. We rewrite $I_{m}\left(R_{m} w, \mu, \Gamma_{\lambda}\right)$ as

$$
I_{m}\left(R_{m} w, \mu, \Gamma_{\lambda}\right)=(1-\mu) \Gamma_{\lambda}\left(R_{m} w\right)-t_{m+1}\left(R_{m} w, \Gamma_{\lambda}\right) .
$$

Since the asymptotic behavior of $\Gamma_{\lambda}\left(R_{m} w\right)$ is known (see (4.9)), then the problem of finding the asymptotic behavior of $I_{m}\left(R_{m} w, \mu, \Gamma_{\lambda}\right)$ is reduced to the problem of finding the asymptotic behavior of $t_{m+1}\left(R_{m} w, \Gamma_{\lambda}\right)$.

Suppose that $w \in \Delta \cap\{w:|w| \leq C\}$ for some constant $C$. By Cauchy's integral formula,

$$
t_{m+1}\left(R_{m} w, \Gamma_{\lambda}\right)=\frac{R_{m}^{m+1} w^{m+1}}{2 \pi i} \oint_{|\xi|=2 R_{m}|w|} \frac{\Gamma_{\lambda}(\xi)}{\xi^{m+1}\left(\xi-R_{m} w\right)} d \xi .
$$

Since

$$
\frac{1}{\xi-R_{m} w}=-\frac{1}{R_{m} w}+\frac{\xi}{R_{m} w\left(\xi-R_{m} w\right)}
$$

then

$$
\begin{aligned}
t_{m+1}\left(R_{m} w, \Gamma_{\lambda}\right) & =-\frac{R_{m}^{m} w^{m}}{2 \pi i} \oint_{|\xi|=2 R_{m}|w|} \frac{\Gamma_{\lambda}(\xi)}{\xi^{m+1}} d \xi+\frac{R_{m}^{m} w^{m}}{2 \pi i} \oint_{|\xi|=2 R_{m}|w|} \frac{\Gamma_{\lambda}(\xi)}{\xi^{m}\left(\xi-R_{m} w\right)} d \xi \\
& =: A_{1}+\frac{R_{m}^{m} w^{m}}{2 \pi i} \oint_{|\xi|=2 R_{m}|w|} \frac{\Gamma_{\lambda}(\xi)}{\xi^{m}\left(\xi-R_{m} w\right)} d \xi
\end{aligned}
$$

where, due to (4.10),

$$
A_{1}=-a_{m} R_{m}^{m} w^{m}=-\frac{w^{m} e^{\frac{m}{\lambda}}}{(2 \pi)^{\frac{1}{2}}}\left(\frac{\lambda^{1-\lambda} e}{2 m^{1+\lambda} \sin (\pi \lambda)}\right)^{\frac{1}{2 \lambda}}(1+o(1)), \quad m \longrightarrow \infty .
$$

Further, we study separately two different cases:

Case 1): $w \in G_{1}=\left\{|w|>1-\frac{\delta}{2}, \quad|w-1|>\delta\right\} \cap \Delta$

Case 2): $w \in G_{2}=\left\{|w| \leq 1-\frac{\delta}{4}, \quad|w-1|>\delta\right\} \cap \Delta$.

Note that $G_{1} \cap G_{2}=\mathbb{C} \cap \Delta \cap\{w:|w-1|>\delta\}$.

Case 1). Suppose that $w \in G_{1}$. By (5.2),

$$
\begin{aligned}
t_{m+1}\left(R_{m} w, \Gamma_{\lambda}\right) & =A_{1}+\frac{R_{m}^{m} w^{m}}{2 \pi i} \oint_{|\xi|=R_{m}|w| / 2} \frac{\Gamma_{\lambda}(\xi)}{\xi^{m}\left(\xi-R_{m} w\right)} d \xi+\Gamma_{\lambda}\left(R_{m} w\right) \\
& =: A_{1}+A_{2}+A_{3}
\end{aligned}
$$

where

$$
A_{2}=\frac{R_{m}^{m} w^{m}}{2 \pi i} \oint_{|\xi|=R_{m}|w| / 2} \frac{\Gamma_{\lambda}(\xi)}{\xi^{m}\left(\xi-R_{m} w\right)} d \xi, \quad A_{3}=\Gamma_{\lambda}\left(R_{m} w\right) .
$$

It follows from (4.9), as $m \rightarrow \infty$, that

$$
\begin{aligned}
A_{3} & =\frac{e^{\frac{m w^{\lambda}}{\lambda}}}{m^{\frac{1}{2 \lambda}}}\left(\frac{\lambda e^{w^{\lambda}}}{2 w^{\lambda} \sin (\pi \lambda)}\right)^{\frac{1}{2 \lambda}}(1+o(1)) \\
& =\frac{w^{m} e^{\frac{m}{\lambda}} e^{\frac{m}{\lambda}\left(w^{\lambda}-\lambda \ln w-1\right)}}{m^{\frac{1}{2 \lambda}}}\left(\frac{\lambda e^{w^{\lambda}}}{2 w^{\lambda} \sin (\pi \lambda)}\right)^{\frac{1}{2 \lambda}}(1+o(1)) .
\end{aligned}
$$

We will find the asymptotic expression for the integral $A_{2}$ in in the following three steps: 
Step 1): change the contour of integration of $A_{2}$;

Step 2): show that the main contribution to $A_{2}$ comes from the neighborhood of the point $\zeta=R_{m}$.

Step 3): find an asymptotic expression for $A_{2}$ by using Laplace's Method for contour integrals.

Consider the curve (see Fig. 5)

$$
T(\lambda)=\left\{z=r e^{i \phi}: r^{\lambda}=\frac{\lambda \phi}{\sin (\lambda \phi)},|\phi| \leq \pi\right\}
$$

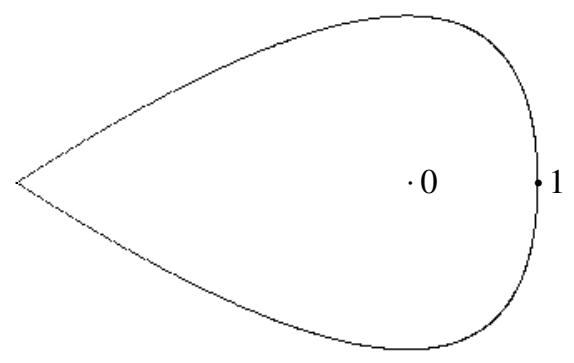

Fig. 5 Curve $T(\lambda)$

Case 1, Step 1. Curves $S\left(\lambda,-\frac{h}{2}\right)$ and $T(\lambda)$ have two points of intersection, say $z_{1}$ and $z_{2}$. We have, $z_{1}=d e^{i \gamma}$ and $z_{2}=d e^{-i \gamma}$, where $\gamma \sim \frac{\sqrt{h}}{\lambda}$ and $d^{\lambda} \sim \frac{\sqrt{h}}{\sin \sqrt{h}}$, as $h \rightarrow 0$. Define

$l_{1}=T(\lambda) \cap\{z:|z| \leq d\}$,

$l_{2}=S\left(\lambda,-\frac{h}{2}\right) \cap\{z:|z| \leq d\}$,

For sufficiently small positive $h$, we have

$$
\begin{aligned}
A_{2} & =\frac{R_{m}^{m} w^{m}}{2 \pi i} \oint_{|\xi|=R_{m}(1-\delta / 2)} \frac{\Gamma_{\lambda}(\xi)}{\xi^{m}\left(\xi-R_{m} w\right)} d \xi \\
& =\frac{R_{m}^{m} w^{m}}{2 \pi i} \oint_{R_{m} l_{1} \cup R_{m} l_{2}} \frac{\Gamma_{\lambda}(\xi)}{\xi^{m}\left(\xi-R_{m} w\right)} d \xi \\
& =\frac{w^{m}}{2 \pi i} \oint_{l_{1} \cup l_{2}} \frac{\Gamma_{\lambda}\left(R_{m} t\right)}{t^{m}(t-w)} d t \\
& =\frac{w^{m}}{2 \pi i}\left(\int_{l_{1}}+\int_{l_{2}}\right) \frac{\Gamma_{\lambda}\left(R_{m} t\right)}{t^{m}(t-w)} d t \\
& =: A_{21}+A_{22},
\end{aligned}
$$

where

$$
A_{21}=\frac{w^{m}}{2 \pi i} \int_{l_{1}} \frac{\Gamma_{\lambda}\left(R_{m} t\right)}{t^{m}(t-w)} d t \quad \text { and } \quad A_{22}=\frac{w^{m}}{2 \pi i} \int_{l_{2}} \frac{\Gamma_{\lambda}\left(R_{m} t\right)}{t^{m}(t-w)} d t
$$

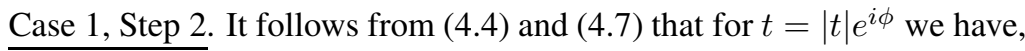

$$
\begin{aligned}
\left|\Gamma_{\lambda}\left(R_{m} t\right)\right| & =\left|\Gamma_{\lambda}\left(R_{m}|t| e^{i \phi}\right)\right| \leq e^{\left(\frac{\pi \cos \lambda \phi}{\sin \pi \lambda}+\alpha\right) R_{m}^{\lambda}|t|^{\lambda}} \\
& =e^{\left(\frac{\pi \cos \lambda \phi}{\sin \pi \lambda}+\alpha\right)\left(\frac{m \sin \pi \lambda}{\pi \lambda}+\frac{\sin \pi \lambda}{2 \pi \lambda}+o(1)\right)|t|^{\lambda}}=e^{\frac{m}{\lambda}(\cos \lambda \phi+\beta)|t|^{\lambda}}
\end{aligned}
$$


where $\beta$ is sufficiently small, $m \geq m(\beta)$. Therefore, since $\Re\left(t^{\lambda}-\lambda \ln t-1\right)=-\frac{h}{2}$ for $t \in l_{2}$, we have

$$
\begin{aligned}
\left|A_{22}\right| & =\left|\frac{w^{m}}{2 \pi i} \int_{l_{2}} \frac{\Gamma_{\lambda}\left(R_{m} t\right)}{t^{m}(t-w)} d t\right| \\
& \leq \text { Const }|w|^{m} \int_{l_{2}} e^{\frac{m}{\lambda}\left(|t|^{\lambda} \cos \lambda \phi-\lambda \ln |t|+\beta|t|^{\lambda}\right)} d|t| \\
& =|w|^{m} O\left(e^{\frac{m}{\lambda}\left(1-\frac{h}{4}\right)}\right) .
\end{aligned}
$$

Case 1, Step 3. The estimation of $A_{21}$ is more complicated than that of $A_{22}$. By (4.9),

$$
\frac{\Gamma_{\lambda}\left(R_{m} t\right)}{t^{m}}=\frac{e^{\frac{m}{\lambda}} e^{\frac{m}{\lambda}\left(t^{\lambda}-\lambda \ln t-1\right)}}{m^{\frac{1}{2 \lambda}}}\left(\frac{\lambda e^{t^{\lambda}}}{2 t^{\lambda} \sin (\pi \lambda)}\right)^{\frac{1}{2 \lambda}}(1+o(1)), \quad m \longrightarrow \infty
$$

where $t \in \Delta$. Thus, since $\Im\left(t^{\lambda}-\lambda \ln t-1\right)=0$ for $t \in l_{1}$ we have

$$
A_{21}=\frac{w^{m} e^{\frac{m}{\lambda}} \lambda^{1 /(2 \lambda)}}{2 \pi i(2 \sin (\pi \lambda) m)^{1 /(2 \lambda)}} \int_{l_{1}}\left(\frac{e^{t^{\lambda}}}{t^{\lambda}}\right)^{1 /(2 \lambda)} \frac{e^{\frac{m}{\lambda}\left(|t|^{\lambda} \cos (\lambda \arg t)-\lambda \ln |t|-1\right)}(1+o(1))}{t-w} d t
$$

Further we use the following lemma.

Lemma 5.1 Suppose that $|w-1| \geq \delta$ and let $p(t)$ be analytic in some neighborhood of $t=1$. Then for sufficiently small positive $h$,

$$
\int_{l_{1}} \frac{e^{\frac{m}{\lambda}\left(t^{\lambda}-\lambda \ln t-1\right)} p(t)}{t-w} d t=\frac{i \sqrt{2 \pi} p(1)(1+o(1))}{\lambda^{\frac{1}{2}}(1-w) m^{1 / 2}}, \quad m \longrightarrow \infty .
$$

Proof. Note that the function $v=-t^{\lambda}+\lambda \ln t+1$ maps the region

$$
\{t:|t-1|<1 / 2\} \cap\left\{t:|t|^{\lambda} \sin (\lambda \arg (t))-\lambda \arg (t)>0\right\}
$$

conformally onto some neighborhood of 0 in the $v$-plane cut along the positive ray. Denote this neighborhood by $U$. In particular, the image of the curve $l_{1}$ is the segment $\left[0, \frac{h}{2}\right]$ traced twice, since

$$
\Im\left(t^{\lambda}-\lambda \ln t-1\right)=0
$$

for $t \in l_{1}$ and the end points $z_{i}, i=1,2$, of $l_{1}$ satisfy the condition $\Re\left(z_{i}^{\lambda}-\lambda \ln z_{i}-1\right)=-\frac{h}{2}, i=1,2$. Rewrite

$$
\int_{l_{1}} \frac{e^{\frac{m}{\lambda}\left(t^{\lambda}-\lambda \ln t-1\right)} p(t)}{t-w} d t=\int_{\mathcal{D}} e^{-\frac{m}{\lambda} v} f(v) d v
$$

where $v=-t^{\lambda}+\lambda \ln t+1, f(v) d v=\frac{p(t)}{t-w} d t$, or equivalently, $f(v)=\frac{t p(t)}{\lambda(t-w)\left(1-t^{\lambda}\right)}$, and $\mathcal{D}=\mathcal{D}_{1} \cup \mathcal{D}_{2}$, where $\mathcal{D}_{1}$ is the upper side of the segment $[0 ; h / 2]$ following the direction of the decrease of $v$ and $\mathcal{D}_{2}$ is the lower side of the segment $[0 ; h / 2]$ following the direction of the increase of $v$.

The transformation $\chi=\sqrt{v}$ maps $U$ onto $\{\chi: \operatorname{Im} \chi>0\} \cap V$ for some neighborhood $V$ of the origin. We have

$$
\chi^{2}=-t^{\lambda}+\lambda \ln t+1=-\frac{\lambda^{2}}{2}(t-1)^{2} \psi(t)
$$


where $\psi(t)$ is an analytic function in some neighborhood of $t=1$ and $\psi(1)=1$. Then

$$
\chi=\frac{\lambda}{\sqrt{2}} i(t-1) \psi_{1}(t)
$$

where $\psi_{1}(t)$ is an analytic function in some neighborhood of $t=1$ and $\psi_{1}(1)=1$. Since $\chi$ is analytic in a neighborhood of $t=1$ and $\chi^{\prime}(1)=\frac{\lambda i}{\sqrt{2}} \neq 0$, the inverse function $t(\chi)$ is analytic in a neighborhood of $\chi=0$, and hence the following function

$$
g(\chi):=\chi f\left(\chi^{2}\right)=\frac{i(t-1) \psi_{1}(t) t p(t)}{\sqrt{2}(t-w)\left(1-t^{\lambda}\right)}=-\frac{i \psi_{1}(t) t p(t)}{\sqrt{2} \lambda(t-w)}(1+o(1)), \quad|t| \longrightarrow 1,
$$

is analytic in some neighborhood of $\chi=0$, say $|\chi| \leq C$, where $C$ is a constant not depending on $w$. If $|\chi|<C / 2$, then

$$
\begin{aligned}
g(\chi) & =\frac{1}{2 \pi i} \int_{|\zeta|=C} \frac{g(\zeta)}{\zeta-\chi} d \zeta \\
& =\frac{1}{2 \pi i} \int_{|\zeta|=C} \frac{g(\zeta)}{\zeta} d \zeta+\frac{\chi}{2 \pi i} \int_{|\zeta|=C} \frac{g(\zeta)}{\zeta(\zeta-\chi)} d \zeta \\
& =g(0)+\chi \alpha(\chi) \\
& =-\frac{i p(1)}{\sqrt{2} \lambda(1-w)}+\chi \alpha(\chi),
\end{aligned}
$$

where $\alpha(\chi)$ is a function analytic in $|\chi|<C / 2$, and

$$
|\alpha(\chi)| \leq \frac{2 \pi C \max _{|\zeta|=C}|g(\zeta)|}{2 \pi C^{2} / 2} \leq C_{3}
$$

where $C_{3}$ is a constant. This implies that $f(v)=g(0) v^{-1 / 2}+\alpha\left(v^{1 / 2}\right)$ in some neighborhood of $v=0$ cut along the positive ray. Let $h$ be so small that $h / 2<\frac{C^{4}}{16}$. Then

$$
\int_{\mathcal{D}} e^{-\frac{m}{\lambda} v} f(v) d v=g(0) \int_{\mathcal{D}} e^{-\frac{m}{\lambda} v} v^{-1 / 2} d v+\int_{\mathcal{D}} e^{-\frac{m}{\lambda} v} \alpha\left(v^{1 / 2}\right) d v=: g(0) J_{1}+J_{2} .
$$

Note that

$$
J_{2}=\int_{\mathcal{D}} e^{-\frac{m}{\lambda} v} \alpha\left(v^{1 / 2}\right) d v=O\left(\frac{1}{m}\right), \quad m \longrightarrow \infty
$$

and

$$
J_{1}=\int_{\mathcal{D}} e^{-\frac{m}{\lambda} v} v^{-1 / 2} d v=\frac{1}{(m / \lambda)^{1 / 2}} \int_{\frac{m}{\lambda} \mathcal{D}} e^{-u} u^{-1 / 2} d u=-\frac{2 \Gamma\left(\frac{1}{2}\right)(1+o(1))}{(m / \lambda)^{1 / 2}}, \quad m \longrightarrow \infty .
$$

Thus,

$$
\int_{\mathcal{D}} e^{-\frac{m}{\lambda} v} f(v) d v=\frac{i \sqrt{2 \pi} p(1)(1+o(1))}{\lambda(1-w)\left(\frac{m}{\lambda}\right)^{1 / 2}}, \quad m \longrightarrow \infty
$$


It follows from (5.10) and Lemma 5.1 that as $w \in G_{1}$,

$$
A_{21}=\frac{w^{m} e^{\frac{m}{\lambda}}}{(2 \pi)^{1 / 2}(1-w)}\left(\frac{\lambda^{1-\lambda} e}{2 m^{\lambda+1} \sin (\pi \lambda)}\right)^{1 /(2 \lambda)}(1+o(1)), \quad m \longrightarrow \infty .
$$

Therefore, by (5.7), (5.9) and (5.11), as $w \in G_{1}$,

$$
A_{2}=\frac{w^{m} e^{\frac{m}{\lambda}}}{(2 \pi)^{1 / 2}(1-w)}\left(\frac{\lambda^{1-\lambda} e}{2 m^{\lambda+1} \sin (\pi \lambda)}\right)^{1 /(2 \lambda)}(1+o(1)), \quad m \longrightarrow \infty
$$

and hence, due to (5.4), (5.3), (5.6), as $w \in G_{1}$

$$
\begin{aligned}
t_{m+1}\left(R_{m} w, \Gamma_{\lambda}\right)= & A_{1}+A_{2}+A_{3} \\
= & \frac{w^{m+1} e^{\frac{m}{\lambda}}}{(2 \pi)^{1 / 2}(1-w)}\left(\frac{\lambda^{1-\lambda} e}{2 m^{\lambda+1} \sin (\pi \lambda)}\right)^{1 /(2 \lambda)}(1+o(1)) \\
& +\frac{w^{m} e^{\frac{m}{\lambda}} e^{\frac{m}{\lambda}\left(w^{\lambda}-\lambda \ln w-1\right)}}{m^{\frac{1}{2 \lambda}}}\left(\frac{\lambda e^{w^{\lambda}}}{2 w^{\lambda} \sin (\pi \lambda)}\right)^{\frac{1}{2 \lambda}}(1+o(1)), \quad m \longrightarrow \infty .
\end{aligned}
$$

It follows from (5.1), (4.9) and (5.12) that

$$
I_{m}\left(R_{m} w, \mu, \Gamma_{\lambda}\right)= \begin{cases}-\frac{w^{m+1} e^{\frac{m}{\lambda}}}{(2 \pi)^{1 / 2}(1-w)}\left(\frac{\lambda^{1-\lambda} e}{2 m^{\lambda+1} \sin (\pi \lambda)}\right)^{1 /(2 \lambda)}(1+o(1)), & w \in I I I_{h} \cap G_{1} ; \\ -\mu \frac{w^{m} e^{\frac{m}{\lambda}} e^{\frac{m}{\lambda}\left(w^{\lambda}-\lambda \ln w-1\right)}}{m^{\frac{1}{2 \lambda}}}\left(\frac{\lambda e^{w^{\lambda}}}{2 w^{\lambda} \sin (\pi \lambda)}\right)^{\frac{1}{2 \lambda}}(1+o(1)), \quad w \in I I_{0} .\end{cases}
$$

Case 2). Suppose that $w \in G_{2}$. By (5.2),

$$
\begin{aligned}
t_{m+1}\left(R_{m} w, \Gamma_{\lambda}\right) & =A_{1}+\frac{R_{m}^{m} w^{m}}{2 \pi i} \oint_{|\xi|=2 R_{m}|w|} \frac{\Gamma_{\lambda}(\xi)}{\xi^{m}\left(\xi-R_{m} w\right)} d \xi \\
& =A_{1}+\frac{w^{m}}{2 \pi i} \oint_{|t|=2|w|} \frac{\Gamma_{\lambda}\left(R_{m} t\right)}{t^{m}(t-w)} d t \\
& :=A_{1}+A_{4} .
\end{aligned}
$$

To find an asymptotic expression for $A_{4}$, we will follow the same three steps that we did to find the asymptotic expression for integral $A_{2}$.

Case 2, Step 1. Note that the curve $S\left(\lambda,-\frac{h}{2}\right)$ intersects the circle $\left\{z:|z|=1-\frac{\delta}{8}=: d_{2}\right\}$ at two points, say $z_{5}=d_{2} e^{i \gamma_{1}}$ and $z_{6}=d_{2} e^{-i \gamma_{1}}$. We write

$$
l_{3}=S\left(\lambda,-\frac{h}{2}\right) \cap\left\{z: d_{2} \leq|z| \leq d\right\}, \quad l_{4}=\left\{z=d_{2} e^{i \phi}, \gamma_{1} \leq \phi \leq 2 \pi-\gamma_{1}\right\},
$$

where $d$ is the same constant that we introduced while considering the curves $l_{1}$ and $l_{2}$.

We have,

$$
\begin{aligned}
A_{4} & =\frac{w^{m}}{2 \pi i} \oint_{l_{1} \cup l_{3} \cup l_{4}} \frac{\Gamma_{\lambda}\left(R_{m} t\right) d t}{t^{m}(t-w)} \\
& =\frac{w^{m}}{2 \pi i}\left(\int_{l_{1}}+\int_{l_{3}}+\int_{l_{4}}\right) \frac{\Gamma_{\lambda}\left(R_{m} t\right) d t}{t^{m}(t-w)} \\
& =: A_{21}+A_{43}+A_{44},
\end{aligned}
$$


where

$$
A_{43}=\frac{w^{m}}{2 \pi i} \int_{l_{3}} \frac{\Gamma_{\lambda}\left(R_{m} t\right) d t}{t^{m}(t-w)} \quad \text { and } \quad A_{44}=\frac{w^{m}}{2 \pi i} \int_{l_{4}} \frac{\Gamma_{\lambda}\left(R_{m} t\right) d t}{t^{m}(t-w)} .
$$

Case 2, Steps 2 and 3. Recall that the asymptotic expression for $A_{21}$ was found in (5.11). The same arguments that we used to estimate integral $A_{22}$ show that

$$
A_{43}=w^{m} o\left(e^{\frac{m}{\lambda}\left(1-\frac{h}{4}\right)}\right), \quad m \longrightarrow \infty .
$$

Using the inequality (5.8), we have

$$
\left|A_{44}\right| \leq \text { Const }|w|^{m} \frac{e^{\frac{m}{\lambda}\left(\cos \lambda \gamma_{1}+\beta\right)\left|1-\frac{\delta}{8}\right|^{\lambda}}}{\left|1-\frac{\delta}{8}\right|^{m}}=|w|^{m} o\left(e^{\frac{m}{\lambda}\left(1-\frac{h}{4}\right)}\right), \quad m \longrightarrow \infty,
$$

for sufficiently small $\delta$ and $h$.

It follows from (5.15), (5.11), (5.16) and (5.17) that as $w \in G_{2}$,

$$
A_{4}=\frac{w^{m} e^{\frac{m}{\lambda}}}{(2 \pi)^{1 / 2}(1-w)}\left(\frac{\lambda^{1-\lambda} e}{2 m^{\lambda+1} \sin (\pi \lambda)}\right)^{1 /(2 \lambda)}(1+o(1)), \quad m \longrightarrow \infty,
$$

and hence, by (5.14), (5.3), (5.18), as $w \in G_{2}$

$$
\begin{aligned}
t_{m+1}\left(R_{m} w, \Gamma_{\lambda}\right) & =A_{1}+A_{4} \\
& =\frac{w^{m+1} e^{\frac{m}{\lambda}}}{(2 \pi)^{1 / 2}(1-w)}\left(\frac{\lambda^{1-\lambda} e}{2 m^{\lambda+1} \sin (\pi \lambda)}\right)^{1 /(2 \lambda)}(1+o(1)), \quad m \longrightarrow \infty .
\end{aligned}
$$

It follows from (5.1), (4.9) and (5.19) that, as $m \rightarrow \infty$,

$$
I_{m}\left(R_{m} w, \mu, \Gamma_{\lambda}\right)= \begin{cases}-\frac{w^{m+1} e^{\frac{m}{\lambda}}}{(2 \pi)^{1 / 2}(1-w)}\left(\frac{\lambda^{1-\lambda} e}{2 m^{\lambda+1} \sin (\pi \lambda)}\right)^{1 /(2 \lambda)}(1+o(1)), & w \in I I I_{h} \cap G_{2} ; \\ (1-\mu) \frac{w^{m} e^{\frac{m}{\lambda}} e^{\frac{m}{\lambda}\left(w^{\lambda}-\lambda \ln w-1\right)}}{m^{\frac{1}{2 \lambda}}}\left(\frac{\lambda e^{w^{\lambda}}}{2 w^{\lambda} \sin (\pi \lambda)}\right)^{\frac{1}{2 \lambda}}(1+o(1)), & w \in I_{0}\end{cases}
$$

Theorem 3.1 is an immediate corollary of (5.13) and (5.20).

It follows from (5.12) and (5.19) that $t_{m+1}\left(R_{m} w, \Gamma_{\lambda}\right)$ does not have zeros in $\{w:|w| \geq 1,|w-1| \geq \delta\}$, as $m \rightarrow \infty$.

\section{Proof of Theorem 3.3}

We suppose that $\xi \in S(0, \lambda)$ and that $|\xi|<1$. By (5.1), (4.9) and (5.19) we have,

$$
\begin{aligned}
I_{m}( & \left.R_{m} \xi\left(1+\varepsilon_{m}(\zeta)\right), \mu, \Gamma_{\lambda}\right) \\
= & (1-\mu) \frac{e^{\frac{m \xi^{\lambda}\left(1+\varepsilon_{m}(\zeta)\right)^{\lambda}}{\lambda}}}{m^{\frac{1}{2 \lambda}}}\left(\frac{\lambda e^{\xi^{\lambda}\left(1+\varepsilon_{m}(\zeta)\right)^{\lambda}}}{2 \xi^{\lambda}\left(1+\varepsilon_{m}(\zeta)\right)^{\lambda} \sin (\pi \lambda)}\right)^{\frac{1}{2 \lambda}}(1+o(1)) \\
& -\frac{\xi\left(1+\varepsilon_{m}(\zeta)\right) \xi^{m}\left(1+\varepsilon_{m}(\xi)\right)^{m} e^{\frac{m}{\lambda}}}{\left(1-\xi-\xi \varepsilon_{m}(\zeta)\right)(2 \pi)^{\frac{1}{2}}}\left(\frac{\lambda^{1-\lambda} e}{2 m^{\lambda+1} \sin (\pi \lambda)}\right)^{\frac{1}{2 \lambda}}(1+o(1)) \\
= & \frac{\xi^{m}\left(1+\varepsilon_{m}(\zeta)\right)^{m} e^{\frac{m}{\lambda}}}{(2 \pi)^{\frac{1}{2}}}\left(\frac{\lambda^{1-\lambda} e}{2 m^{1+\lambda} \sin (\pi \lambda)}\right)^{\frac{1}{2 \lambda}}(1+o(1)) \\
& \times\left((1-\mu) \frac{e^{\frac{m}{\lambda}\left(\xi^{\lambda}\left(1+\varepsilon_{m}(\zeta)\right)^{\lambda}-\lambda \ln \left(\xi\left(1+\varepsilon_{m}(\zeta)\right)-1\right)\right.} m^{\frac{1}{2}} e^{\frac{\xi^{\lambda}\left(1+\varepsilon_{m}(\zeta)\right)^{\lambda}}{2 \lambda}}(2 \pi \lambda)^{\frac{1}{2}}}{\xi^{\frac{1}{2}}\left(1+\varepsilon_{m}(\zeta)\right)^{\frac{1}{2}} e^{\frac{1}{2 \lambda}}} \frac{\xi\left(1+\varepsilon_{m}(\zeta)\right)}{1-\xi-\xi \varepsilon_{m}(\zeta)}(1+o(1))\right) \\
= & \left((1-\mu)\left(\frac{2 \pi \lambda}{\xi}\right)^{\frac{1}{2}} e^{\frac{\xi^{\lambda}-1}{2 \lambda}} e^{\frac{m}{\lambda}\left(\xi^{\lambda}\left(1+\varepsilon_{m}(\zeta)\right)^{\lambda}-\lambda \ln \left(\xi\left(1+\varepsilon_{m}(\zeta)\right)-1+\frac{\lambda \ln m}{2 m}\right)\right.}(1+o(1))-\frac{\xi}{1-\xi}(1+o(1))\right) C,
\end{aligned}
$$


where

$$
C=\frac{\xi^{m}\left(1+\varepsilon_{m}(\zeta)\right)^{m} e^{\frac{m}{\lambda}}}{(2 \pi)^{\frac{1}{2}}}\left(\frac{\lambda^{1-\lambda} e}{2 m^{1+\lambda} \sin (\pi \lambda)}\right)^{\frac{1}{2 \lambda}}
$$

Since $\xi=|\xi| e^{i \phi} \in S(\lambda, 0)$ and $\tau_{m}=\frac{m}{\lambda} \tau+2 \pi k$ for some $k$ in $\mathbb{Z}$, we have

$$
\begin{aligned}
& \xi^{\lambda}\left(1+\varepsilon_{m}(\zeta)\right)^{\lambda}-\lambda \ln \xi-\lambda \ln \left(1+\varepsilon_{m}(\zeta)\right)-1+\frac{\lambda \ln m}{2 m} \\
&=\xi^{\lambda}-\lambda \ln \xi-1+\xi^{\lambda}\left(\left(1+\varepsilon_{m}(\zeta)\right)^{\lambda}-1\right)-\lambda \ln \left(1+\varepsilon_{m}(\zeta)\right)+\frac{\lambda \ln m}{2 m} \\
&= i\left(|\xi|^{\lambda} \sin \lambda \phi-\lambda \phi\right)+\xi^{\lambda}\left(\frac{\lambda \ln m}{2\left(1-\xi^{\lambda}\right) m}-\frac{\lambda \zeta-\lambda i \tau_{m}}{\left(1-\xi^{\lambda}\right) m}+o\left(\frac{1}{m}\right)\right) \\
&-\frac{\lambda \ln m}{2\left(1-\xi^{\lambda}\right) m}+\frac{\lambda \zeta-i \lambda \tau_{m}}{\left(1-\xi^{\lambda}\right) m}+\frac{\lambda \ln m}{2 m} \\
&= \frac{\lambda \zeta}{m}-i \frac{2 \lambda \pi k}{m}+o\left(\frac{1}{m}\right)
\end{aligned}
$$

for some integer $k$. Therefore, for $\xi \in S(\lambda, 0)$ and $|\xi|<1$, as $m \rightarrow \infty$

$$
\frac{I_{m}\left(R_{m} \xi\left(1+\varepsilon_{m}(\zeta)\right), \mu, \Gamma_{\lambda}\right)}{C} \longrightarrow(1-\mu)\left(\frac{2 \pi \lambda}{\xi}\right)^{\frac{1}{2}} e^{\frac{\xi^{\lambda}-1}{2 \lambda}} e^{\zeta}-\frac{\xi}{1-\xi} .
$$

We suppose that $\xi \in S(\lambda, 0)$ and that $|\xi|>1$. Then, by (5.1), (4.9) and (5.12),

$$
\begin{aligned}
I_{m}\left(R_{m} \xi\left(1+\varepsilon_{m}(\zeta)\right), \mu, \Gamma_{\lambda}\right) & \\
= & -\mu \frac{e^{\frac{m \xi^{\lambda}\left(1+\varepsilon_{m}(\zeta)\right)^{\lambda}}{\lambda}}}{m^{\frac{1}{2 \lambda}}}\left(\frac{\lambda e^{\xi^{\lambda}\left(1+\varepsilon_{m}(\zeta)\right)^{\lambda}}}{2 \xi^{\lambda}\left(1+\varepsilon_{m}(\zeta)\right)^{\lambda} \sin (\pi \lambda)}\right)^{\frac{1}{2 \lambda}}(1+o(1)) \\
& \quad-\frac{\xi\left(1+\varepsilon_{m}(\zeta)\right) \xi^{m}}{\left(1-\xi-\xi \varepsilon_{m}(\zeta)\right)(2 \pi)^{\frac{1}{2}}}\left(\frac{\lambda^{1-\lambda} e}{2 m^{\lambda+1} \sin (\pi \lambda)}\right)^{\frac{1}{2 \lambda}}(1+o(1)) .
\end{aligned}
$$

The expression for

$$
I_{m}\left(R_{m} \xi\left(1+\varepsilon_{m}(\zeta)\right), \mu, \Gamma_{\lambda}\right)
$$

with $|\xi|>1$ differs from the expression for

$$
I_{m}\left(R_{m} \xi\left(1+\varepsilon_{m}(\zeta)\right), \mu, \Gamma_{\lambda}\right)
$$

with $|\xi|<1$ only by one coefficient, namely, instead of $(1-\mu)$ we have $\mu$. The same calculations that were done for $I_{m}\left(R_{m} \xi\left(1+\varepsilon_{m}(\zeta)\right), \mu, \Gamma_{\lambda}\right)$ with $|\xi|<1$ show that, as $m \rightarrow \infty$,

$$
\frac{I_{m}\left(R_{m} \xi\left(1+\varepsilon_{m}(\zeta)\right), \mu, \Gamma_{\lambda}\right)}{C} \longrightarrow-\mu\left(\frac{2 \pi \lambda}{\xi}\right)^{\frac{1}{2}} e^{\frac{\xi^{\lambda}-1}{2 \lambda}} e^{\zeta}-\frac{\xi}{1-\xi}
$$

This completes the proof of Theorem 3.3.

\section{Proof of Theorem 3.5}

By (4.11), as $m \rightarrow \infty$,

$$
\ln F\left(R_{m}\left(1+\left(\frac{2}{\lambda m}\right)^{1 / 2} \zeta\right)\right)-\ln F\left(R_{m}\right)=\left(\frac{2}{\lambda m}\right)^{1 / 2} \zeta m+\frac{\zeta^{2}(\lambda-1+o(1))}{\lambda}+o(1) .
$$


Hence,

$$
\ln \frac{F\left(R_{m}\left(1+\left(\frac{2}{\lambda m}\right)^{1 / 2} \zeta\right)\right)}{\left(1+\left(\frac{2}{\lambda m}\right)^{1 / 2} \zeta\right)^{m} F\left(R_{m}\right)}=\zeta^{2}+o(1), \quad m \longrightarrow \infty
$$

and then, by (1.7), as $m \rightarrow \infty$,

$$
\begin{aligned}
& \frac{t_{m+1}\left(R_{m}\left(1+\left(\frac{2}{\lambda m}\right)^{1 / 2} \zeta\right), F\right)}{\left(1+\left(\frac{2}{\lambda m}\right)^{1 / 2} \zeta\right)^{m} F\left(R_{m}\right)} \\
& \quad=\frac{F\left(R_{m}\left(1+\left(\frac{2}{\lambda m}\right)^{1 / 2} \zeta\right)\right)}{\left(1+\left(\frac{2}{\lambda m}\right)^{1 / 2} \zeta\right)^{m} F\left(R_{m}\right)}-\frac{s_{m}\left(R_{m}\left(1+\left(\frac{2}{\lambda m}\right)^{1 / 2} \zeta\right), F\right)}{\left(1+\left(\frac{2}{\lambda m}\right)^{1 / 2} \zeta\right)^{m} F\left(R_{m}\right)} \\
& \quad \longrightarrow \exp \left\{\zeta^{2}\right\}-\frac{1}{2} \exp \left\{\zeta^{2}\right\} \operatorname{erfc}(\zeta)
\end{aligned}
$$

uniformly on every compact set of the $\zeta$-plane. Theorem 3.5 follows immediately from (1.5), (1.7) and (7.1).

\section{References}

[1] J. Dieudonné, Sur les zéros des polynomes-sections de $e^{x}$, Bull. Soc. Math. France 70, 333-351 (1935).

[2] A. Edrei, E. B. Saff, and R. S. Varga, Zeros of sections of power series, Lecture Notes in Math. 1002, 1-115 (1983).

[3] M. A. Evgrafov, Asymptotic Estimates and Entire Functions (Gordon and Breach, Science Publishers, Inc., New York, 1961).

[4] B. Ya. Levin, Distribution of Zeros of Entire Functions (American Mathematical Society, Providence, Rhode Island, 1980).

[5] B. Ya. Levin, Lectures on Entire Functions (American Mathematical Society, Providence, Rhode Island, 1996).

[6] I. V. Ostrovskii, On zero distribution of sections and tails of power series, Isr. Math. Conf. Proc. 15, 297-310 (2001).

[7] P. C. Rosenbloom, On Sequences of Polynomials, Especially Sections of Power Series, Ph.D. thesis, Stanford University (1944).

[8] G. Szegö, Über eine Eigenschaft der Exponentialreihe, Sitzungsber. der Berliner Math. Gesellschaft 23, 50-64 (1924).

[9] N. A. Zheltukhina, Asymptotic distribution of zeros of sections and tails of Mittag-Leffler functions, C. R. Acad. Sci. Paris, Sér I, Math. 335, 133-138 (2002). 